\title{
Nallıhan (Ankara) İlçesi Makrofungusları
}

\author{
Celâleddin ÖZTÜRK, Dilek PAMUKÇU, Sinan AKTAŞ
}

\section{Selçuk Üniversitesi Fen Fakültesi Biyoloji Bölümü, 42075, Selçuklu, Konya}

Öz: Nallıhan (Ankara) İlçesinin farklı lokalitelerinden Mayıs 2003 ve Haziran 2006 yılları arasında makrofungusların yetişmesi için uygun olan mevsimlerde 107 makrofungus örneği toplanmıştır. Arazi ve laboratuvar çalışmaları sonucunda, Ascomycota ve Basidiomycota bölümlerine ait toplam 68 tür tespit edilmiştir. Türlerin tamamı yöre için yeni kayıttır.

Anahtar kelimeler: Makrofungus, Nallıhan, Ankara, Türkiye.

\section{Macrofungi of Nallıhan (Ankara) District}

Abstract: 107 macrofungi samples were collected from different localities of the Nallıhan (Ankara) district between May 2003 and June 2006, which were suitable for the growth of macrofungi. As a result of the field and laboratory studies, 68 species belong to Ascomycota and Basidiomycota divison were identified. All of these species are new records for the region.

Key words: Macrofungus, Nallıhan, Ankara, Türkiye.

\section{Giriş}

Ankara-Eskişehir-Bolu üçgeninin ortasında yer alan Nallıhan, Karadeniz bölgesinin Batı Karadeniz bölümünde yer alır. Ankara'nın batısında ve çok engebeli bir arazi yapısına sahip olan Nallıhan, Doğudan Beypazarı, Kuzeybatıdan Göynük, Kuzeyden MudurnuSeben, Batıdan Sarıcakaya, Güneyden Eskişehir ve Mihalıçcık'la çevrilidir (Şekil 1).

İlçe arazisinin \% 48'i orman, \% 25'i tarım, $\% 22$ 'si çayır ve mera ve $\%$ 5'i çalılık alanlardan oluşmaktadır. Dağları, çam ve meşe ormanları ile kaplı olup kuzey ve batı yönünde orman örtüsü sık, doğu ve güney yönünde ise genellikle çıplak olup Çayırhan ve Beypazarı'na komşu arazileri kısmen düzlük alanlardan oluşmaktadır. Araştırma alanında Köknar, çam, ardıç, yer yer meşe ormanları ve diğer yapraklı ağaçlar görülür. İğne yapraklı ağaçlar yaygın türleri oluşturur. Bunlar, Abies nordmanniana (Stev) Spach. subsp. bornmuelleriana (Mattf) Coode et Cullen., Pinus brutia Ten., Pinus nigra Arnold subsp. pallasiana (Lamb.) Holmboe., Pinus sylvestris L., Juniperus communis L. subsp. nana, Juniperus excelsa Biep., Juniperus oxycedrus L. subsp. oxycedrus. Bölgede yapraklı ağaçları da Acer platonoides L., Acer tataricum L., Corylus avellana L., Eleagnus angustifolia L., Quercus cerris L., Quercus pubescens Willd., Ficus carica L., Prunus cocomilio Ten., Prunus domestica L. subsp. insititia Poir., Pyrus eleagnifolia Pall., Salix alba L. ve Salix purpurea L. temsil eder.

İlçenin iklimi, İç Anadolu ve Batı Karadeniz Bölgeleri iklim özelliklerini göstermektedir. İlkbahar, sonbahar ve kış ayları genellikle yağışı geçerken yaz aylarında pek yağış görülmemektedir. Kış aylarının fazla soğuk olmadığı ilçede, rakımı 200-250 metreye kadar düşen Sakarya Nehri Vadisinde ise daha Ilıman bir iklim tipi görülür.

Ülkemizde makrofunguslar üzerine yapılan çalışmaların, son yıllarda artış gösterdiğini görmekteyiz. 


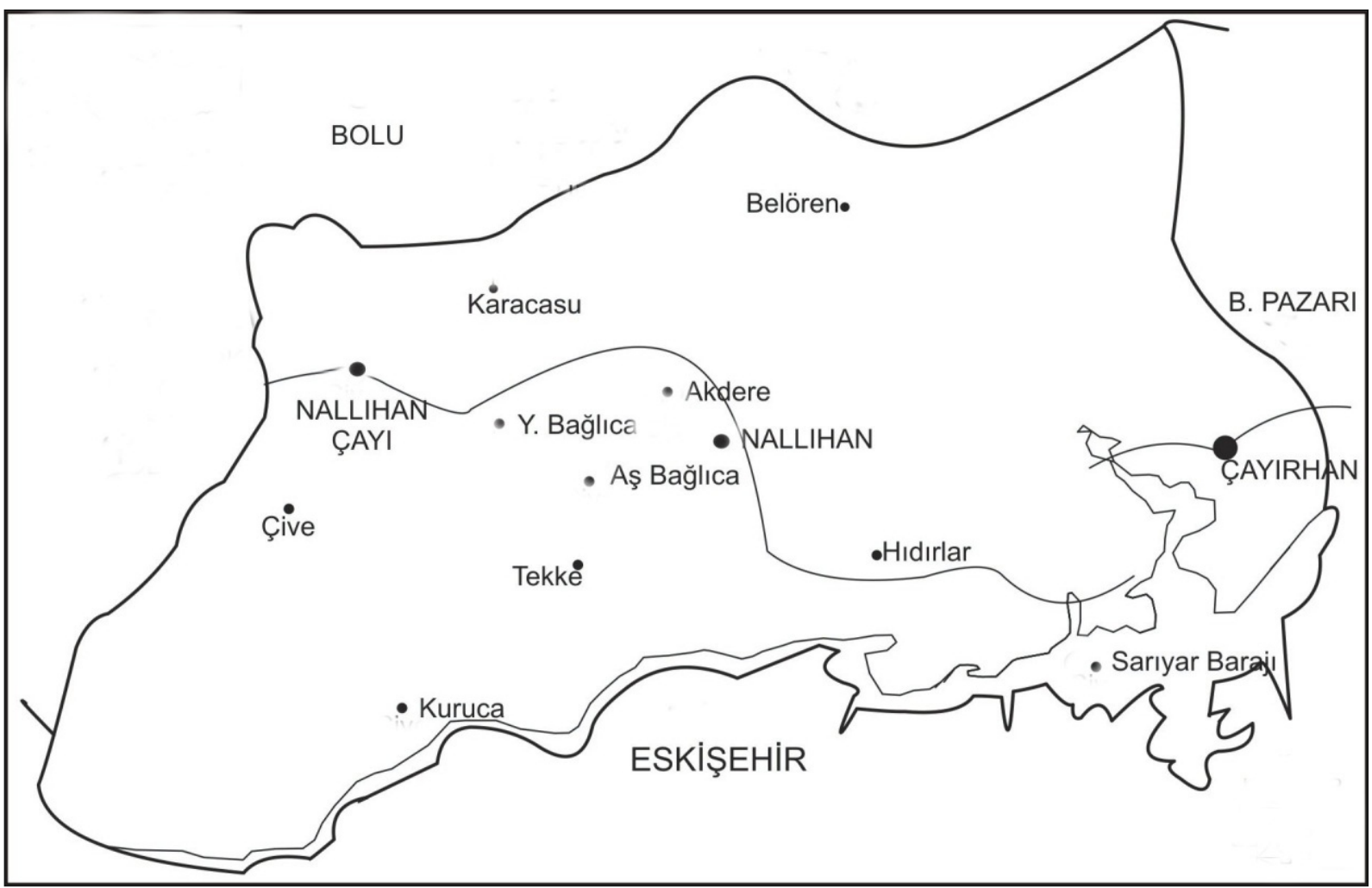

Şekil 1. Araştırma Alanı

Yapılan bu çalışmaların bir kısmı iki ayrı kontrol listesi halinde yayınlanmıştır (Doğan ve Ark., 2007; Sesli ve Denchev, 2014) ve halen devam eden çalışmalar ile katkı yapılmaya devam etmektedir. Yaptığımız bu çalışma ile de Türkiye makrofunguslarına katkı yapılması ve ayrıca belirlenen yenen ve zehirli mantarlar ile bölge halkının bilgilendirilmesi amaçlanmıştır.

\section{Materyal Ve Metot}

Çalışma materyalini Mayıs 2003 ve Haziran 2006 tarihleri arasındaki sonbahar ve ilkbahar aylarında, yağışın bol olduğu zamanlarda toplanan mantar numuneleri oluşturmaktadır. Toplanan numuneler laboratuvara getirilip, mantar örnekleri üzerinde yapılan çalışmaların neticesinde türlerin deskripsiyonu çıkarılmıştır. Elde edilen veriler ve mevcut literatürün yardımıyla türlerin teşhisleri yapılmıştır (Watling
(1973), Philips (1981), Moser (1983), Ellis ve Ellis (1990), Breitenbach ve Krânzlin (1984,1986, 1991, 1995, 2000, 2005), Dähncke (1993),Jordan (1995), Winkler (1996). Mantarlar fungaryum materyali halinde hazırlanırken, kurutma dolabı içinde kurutulmuştur. Kurutulan örnekleri parazitlerden korumak için, içerisine 50 gr kristal timol veya fenol kristali yerleştirilmiş ve $50^{\circ} \mathrm{C}$ ' ye ayarlanmış etüve konularak 3 saat bekletilmiştir. Böylece timol ve fenolün buharlaşarak mantar dokusuna nüfuz etmesi sağlanmıştır. Etüvden çıkarılan mantarlar kilitli polietilen poşetler içine yerleştirilerek, her mantar cinsi kendisi için ayrılmış saklama kutularına konmuştur. Mantar örnekleri S.Ü. Mantarcılık Uygulama ve Araştırma Merkezi Fungaryumu'nda saklanmaktadır. 


\section{Bulgular}

Yapılan arazi ve laboratuvar çalışmaları sonucu tespit edilen mantar türleri aşağıda alfabetik liste (Her bir kategori kendi içinde sıralanmıştır) halinde verilmiştir (Kısaltmalar: DP, Dilek Pamukcu).

\section{Ascomycota \\ Pezizomycetes}

\section{Pezizales}

Helvellaceae

Helvella spadicea Schaeff.

Nallıhan deresi kenarı, kavaklık alan, 14.05.2003, DP 1.

\section{Pezizaceae}

Sarcosphaera coronaria (Jacq.) J. Schröt.

Nallıhan Tv vericisi altı, çam ormanı, 11.06.2004, DP23.

\section{Basidiomycota \\ Agaricomycetes \\ Agaricales}

\section{Agaricaceae}

Agaricus bitorquis (Quél.) Sacc.

Hıdırlar köyü, çay kenarı, çayırlık alan, 15.08.2005, DP 77, 17.06.2004, DP 52. Tekke köyü girişi, çam ormanı, 29.05.2005, DP 55

Agaricus macrocarpus F.H. Møller Hıdırlar köyü, dere kenarı, çayırlık alan, 10.06.2004, DP 19.

\section{Bovista plumbea Pers.}

Nalıhan Tv vericisi altı, çam ormanı, 11.06.2004, DP 24.

Coprinus comatus (O.F. Müll.) Pers.

Nallıhan çayı kenarı, kavak ağacı altı, 14.08.2005, DP 76.

Macrolepiota excoriata (Schaeff.) Wasser

Kuruca köyü girişi, çam ormanı, 25.05.2006, DP 105.

Macrolepiota procera (Scop.) Singer

Tekke köyü, çam ormanı, 21.06.2005, DP 64.

Tekke köyü yaylası, çam ormanı, 19.08.2005,
DP 83.

Amanitaceae

Amanita citrina Pers.

Balca köyü, çam ormanı, 12.6.2005, DP 56

Amanita gemmata (Fr.) Bertill.

Nallıhan Tv vericisi altı, çam ormanı, 11.06.2004, DP 27. Aksu köyü yolu üzeri, çam ormanı, 11.06.2004, DP 29.

Amanita ovoidea (Bull.) Link

Hoşebe mevkii, çam ormanı, 26.05.2006, DP 107. Kuruca köyü girişi, çam ormanı, 10.06.2004, DP 17. Tekke köyü yaylası, çam ormanı, 22.08.2005, DP 85. Balca köyü, çam ormanı, 13.06.2004, DP 41. Tekke köyü yaylası, su deposu aşağısı, çam ormanı, 10.06.2004, DP 12.

Amanita pantherina (DC.) Krombh.

Balca köyü, çam ormanı, 12.06.2005, DP 59.

\section{Cortinariaceae}

Cortinarius flexipes (Pers.) Fr.

Hoşebe mevkii, çam ormanı, 26.05.2006, DP 106.

\section{Cortinarius rubellus Cooke}

Balca köyü, çam ormanı, 12.06.2005, DP 60.

Cortinarius trivialis J.E. Lange

Kuruca köyü, çam ormanı, 25.05.2006, DP 100.

Hymenogastraceae

Hebeloma eburneum Malençon

Aksu köyü, çam ormanı, 12.06.2004, DP 33.

Hebeloma sinapizans (Fr.) Sacc.

Tekke köyü yaylası, çam ormanı, 22.08.2005, DP 88.

Inocybaceae

Inocybe mixtilis (Britzelm.) Sacc.

Belören köyü girişi, çam ormanı, 12.06.2004, DP 31.

Inocybe rimosa (Bull.) P. Kumm.

Kuruca köyü, çam ormanı, 06.06.2004, DP 7.

Omphalotaceae

Gymnopus erythropus (Pers.) Antonín, Halling \& Noordel.

Nallıhan çayı kenarı, meşelik alan, 17.08.2005, DP 80. 
Omphalotus illudens (Schwein.) Bresinsky \& Besl

Balca köyü, meşelik alan, 12.06.2005, DP 61.

\section{Pleurotaceae}

Pleurotus ostreatus (Jacq.) P. Kumm., Balca köyü, kesilmiş kavak ağacı üzeri, 13.06.2004, DP 40.

\section{Pluteaceae}

Volvopluteus gloiocephalus (DC.) Vizzini, Contu \& Justo

Balca köyü, çam ormanı, çayırlık alan, 13.06.2004, DP 35. Kuruca köyü girişi, çam ormanı, çayırlık alan, 25.05.2006, DP 102.

Psathyrellaceae

Coprinellus micaceus (Bull.) Vilgalys, Hopple \& Jacq. Johnson

Hıdırlar köyü, dere kenarı, kavak ağacı altı, 10.06.2004, DP 21.

\section{Psathyrella candolleana (Fr.) Maire}

Çayırhan kasabası, dut ağacı altı, 12.06.2003, DP 3.

Psathyrella cotonea (Quél.) Konrad \& Maubl. Nallıhan çayı kenarı, Akdere köyü altı, çayırlık alan, 14.08.2005, DP 73.

\section{Schizophyllaceae}

Schizophyllum commune Fr.

Nallıhan çayı kenarı, kesilmiş kavak üzeri, 14.08.2005, DP 75. Balca köyü, kesilmiş meyve ağacı üzeri, 13.06.2004, DP 36.

\section{Strophariaceae}

Agrocybe dura (Bolton) Singer

Balca köyü yol kenarı, çayırlık alan, 13.06.2004, DP 38.

Agrocybe praecox (Pers.) Fayod

Nallıhan çayı kenarı, çayırlık alan, 15.05.2003, DP2.

Cyclocybe cylindracea (DC.) Vizzini \& Angelini Nallıhan çayı kenarı, kavak kütüğü üzeri, 14.08.2005, DP 72. Nallıhan çayı kenarı, Akdere köyü altı, kavak kütüğü üzeri, 14.08.2005, DP 67.

\section{Tricholomataceae}

Clitocybe dealbata (Sowerby) P. Kumm.
Kuruca köyü girişi, çam ormanı, 25.05.2006, DP 92.

Lepista nuda (Bull.) Cooke

Kuruca köyü girişi, çam ormanı, 25.05.2006, DP 99.

\section{Tricholoma batschii Gulden}

Tekke köyü yaylası, çam ormanı, 10.06.2004, DP 15

\section{Tricholoma bresadolanum Clémençon}

Kuruca köyü girişi, çam ormanı, 25.05.2006, DP 101.

Tricholoma imbricatum (Fr.) P. Kumm.

Nallıhan Tv vericisi altı, çam ormanı, 11.06.2004, DP 28. Beleören köyü, yol üzeri, çam ormanı, 15.06.2004, DP 47. Tekke köyü yayla girişi, çam ormanı, 10.06.2004, DP 16, 22.08.2005, DP 87. Balca köyü, çam ormanı, 12.06.2005, DP 57.

Tricholoma myomyces (Pers.) J.E. Lange Kuruca köyü, çam ormanı, 06.06.2004, DP 9. Nalıhan Tv vericisi altı, çam ormanı, 10.06.2004, DP 11. Kuruca köyü girişi, yol kenarı, çam ormanı, 25.05.2006, DP 103.

Tricholoma scalpturatum (Fr.) Quél.

Kuruca köyü, çam ormanı, 06.06.2004, DP 6.

\section{Boletales}

Boletaceae

Boletus erythropus Krombh.

Balca köyü, çam ormanı, 14.06.2004, DP 43.

\section{Boletus impolitus Fr.}

Tekke köyü yaylası, çam ormanı, 19.08.2005, DP 81.

Boletus luridus Schaeff.

Balca köyü girişi, çam ormanı, 12.06.2005, DP 58.

\section{Boletus speciosus Frost}

Kuruca köyü, çam ormanı, 10.06.2004, DP 18.

\section{Boletus subtomentosus $\mathrm{L}$.}

Tekke köyü yaylası, çam ormanı, 10.06.2004, DP 20.

Leccinellum crocipodium (Letell.) Della Maggiora \& Trassin

Balca köyü, çam ormanı, 17.06.2005, DP 62. 


\section{Gomphidiaceae}

Chroogomphus rutilus (Schaeff.) O.K. Mill.

Kuruca köyü, çam ormanı, 06.06.2004, DP 8. Nallıhan Tv vericisi altı, çam ormanı, 11.06.2004, DP 25. Balca köyü, çam ormanı, 13.06.2004, DP 37. Kuruca köyü girişi, çam ormanı, 25.05.2006, DP 95.

\section{Rhizopogonaceae}

Rhizopogon roseolus (Corda) Th. Fr.

Belören köyü, çam ormanı, 12.06.2004, DP 32, 15.06.2004, DP 45. Aşağı Düşen köyü, çam ormanı, 12.06.2004, DP 34. Belören köyü, çam ormanı,. Aşağı Belören köyü, çam ormanı, 15.06.2004, DP 51.

\section{Suillaceae}

Suillus luteus (L.) Roussel

Aksu köyü yaylası, yol kenarı, çam ormanı, 20.05.2006, DP 90. Kuruca köyü girişi, çam ormanı, 25.05.2006, DP 98.

\section{Geastrales}

\section{Geastraceae}

Geastrum triplex Jungh.

Aksu köyü yaylası, çam ormanı, 20.05.2006, DP 89.

\section{Hymenochaetales}

Hymenochaetaceae

Inocutis rheades (Pers.) Fiasson \& Niemelä

Nallıhan çayı kenarı, Akdere köyü altı, kesilmiş kavak üzeri, 14.08.2005, DP 74. Nallıhan çayı kenarı, yüzme havuzu arkası, kesilmiş kavak üzeri, 17.08.2005, DP 78. Belören köyü yol üzeri, kesilmiş kavak ağacı üzeri, 15.06.2004, DP 50.

Porodaedalea pini (Brot.) Murrill

Kuruca köyü girişi, çam ağacı üzeri, 25.05.2006, DP 104.

Phellinus pomaceus (Pers.) Maire

Kuruca köyü girişi, çam ormanı, 25.05.2006, DP 94.

\section{Polyporales}

Fomitopsidaceae
Laetiporus sulphureus (Bull.) Murrill

Nallıhan çayı kenarı, stadyum arkası, söğüt ağacı üzeri, 26.05.2005, DP 54.

\section{Meruliaceae}

Bjerkandera adusta (Willd.) P. Karst.

Nallıhan çayı kenarı, kavak kütüğü üzeri, 14.08.2005, DP 65. Yüzme havuzu arkası, kavak kütüğü üzeri, 14.08.2005, DP 68.

\section{Polyporaceae}

Cerioporus squamosus (Huds.) Quél

Nallıhan-Bolu yolu üzeri yol kenarı, dut ağacı üzeri, 21.05.2006, DP 91.

Fomes fomentarius (L.) Gillet

Nallıhan çayı kenarı, kavak ağacı üzeri, 14.08.2005, DP 69. Balca köyü, kavak ağacı üzeri, 17.06.2005, DP63.

Lentinus brumalis (Pers.) Zmitr.

Balca köyü, çam ormanı, ağaç üzeri, 13.06.2004, DP 39.

Panus conchatus (Bull.) Fr.

Nallıhan çayı kenarı, kavak ağacı üzeri, 14.08.2005, DP 66.

Trametes versicolor (L.) Lloyd

Tekke köyü yaylası, meyve ağacı üzeri, 17.08.2005, DP 79.

\section{Russulales}

\section{Auriscalpiaceae}

Lentinellus cochleatus (Pers.) P. Karst.

Nallıhan çayı kenarı, kesilmiş kavak ağacı üzeri, 14.08.2005, DP 70.

\section{Russulaceae}

Lactarius deliciosus (L.) Gray

Nallıhan Tv vericisi altı, çam ormanı, 11.06.2004, DP 26.

Lactarius vellereus (Fr.) Fr.

Belenören köyü yolu, çam ormanı, 15.06.2004, DP 48.

Russula emetica (Schaeff.) Pers.

Tekke köyü yaylası, çam ormanı, 19.08.2005, DP 82. Aşağı Çive köyü, çam-meşe ormanı, 26.06.2004, DP 53. 


\section{Russula nigricans Fr.}

Tekke köyü yaylası, çam ormanı, 19.08.2005, DP 84.

\section{Russula ochroleuca Fr.}

Hoşebe piknik alanı, çam ormanı, 11.06.2004, DP 22. Tekke köyü yaylası, çam ormanı, 10.06.2004, DP 13. Balca köyü, çam ormanı, 14.06.2004, DP 42

\section{Stereaceae}

Stereum hirsutum (Willd.) Pers.

Belören köyü, yol üzeri, çam ormanı, 15.06.2004, DP 49.

Stereum ochraceoflavum (Schwein.) Sacc. Tekke köyü yaylası, kesilmiş meyve ağacı üzeri, 22.08.2005, DP 86.

\section{Thelephorales}

Bankeraceae

Boletopsis leucomelaena (Pers.) Fayod

Kuruca köyü girişi, çam ormanı, 25.05.2006, DP 97.

\section{Phellodon tomentosus (L.) Banker}

Karacasu köyü, şelale yolu üzeri, çam ormanı, 14.08.2005, DP71.

\section{Thelephoraceae}

Thelephora caryophyllea (Schaeff.) Pers. Aşağı düşen köyü, çam ormanı, 14.06.2004, DP 44.

\section{Tartışma}

2003-2006 yılları arasında yapılan arazi ve laboratuar çalışmaları sonucunda iki bölüm, sekiz takım, 28 familya, 45 cinse ait 68 tür tespit edilmiştir. Bu türlerden iki tanesi Ascomycota, 66 tanesi de Basidiomycota bölümüne aittir. Türlerin familyalara göre dağılımı şöyledir; : Tricholomataceae 7, Agaricaceae ve Boletaceae 6, Polyporaceae ve Russulaceae 5, Amani- taceae 4, Strophariaceae, Cortinariaceae, Psathyrellaceae ve Hymenochaetaceae 3, Inocybaceae, Omphalotaceae, Hymenogastraceae, Stereaceae ve Bankeraceae 2, Helvellaceae, Pezizaceae, Pleurotaceae, Pluteaceae, Schizophyllaceae, Gomphidiaceae, Rhizopogonaceae, Suillaceae, Geastraceae, Meruliaceae, Fomitopsidaceae, Auriscalpiaceae ve Thelephoraceae 1.

Çalışma alanı ve yakın çevresinde yapılan çalışmalar karşılaştıııldığında (Tablo 1), oransal olarak bakıldığında Tricholomataceae, Agaricaceae, Polyporaceae ve Russulaceae familyalarına ait türlerin yoğunluğunu görmekteyiz. Çalışma alanında Ascomycota bölümüne ait tür sayısının diğer çalışmalara göre az sayıda olduğu, Boletaceae ve Amanitaceae familyalarına ait türlerin karışık ormanların varlığından dolayı sayıca fazlalığı görülmektedir.

Yapılan çalışma ile bölgede belirlenen yenen türler: Helvella spadicea, Agaricus bitorquis, A. macrocarpus, Bovista plumbea, Coprinus comatus, Macrolepiota excoriata, M. procera, Pleurotus ostreatus, Volvopluteus gloiocephalus, Coprinellus micaceus, Lepista nuda, Leccinellum crocipodium, Chroogomphus rutilus, Rhizopogon roseolus, Lactarius deliciosus ve Russula ochroleuca'dir.

Sonuç olarak bakıldığında, bölgede tespit edilen yenen türler, toplam türlerin \% 23.5 ini, zehirli türler \% 20.6 sını oluşturmaktadır. Belirlenen bu türler ile halkın yenen ve zehirli türler hakkında bilinçlendirilmesi sağlanmıştır. Yapılacak benzer çalışmalar ile hem halkın sağııkı bir besin kaynağı olan mantarlara ilgisi, hem de bilgisinin artırılacağı kanaatindeyiz. 
Tablo 1. Çalışma alanı ve çalışma alanına yakın çalışmaların familyalara göre dağılımı

\begin{tabular}{|c|c|c|c|c|c|c|}
\hline & $\begin{array}{l}\text { Servi ve } \\
\text { Ark. } \\
\text { (2010) }\end{array}$ & $\begin{array}{l}\text { Akata ve } \\
\text { Ark. } \\
(2010)\end{array}$ & $\begin{array}{l}\text { Kaşık } \\
\text { ve Ark. } \\
\text { (2010) }\end{array}$ & $\begin{array}{l}\text { Akata ve } \\
\text { Ark. } \\
\text { (2009) }\end{array}$ & $\begin{array}{l}\text { Köstekçi } \\
\text { ve Ark. } \\
\text { (2005) }\end{array}$ & $\begin{array}{l}\text { Çalışma } \\
\text { alanı }\end{array}$ \\
\hline Helotiaceae & 1 & 4 & 1 & 1 & - & - \\
\hline Helvellaceae & - & - & 2 & 1 & 4 & 1 \\
\hline Humariaceae & - & - & - & - & 1 & - \\
\hline Hyaloscyphaceae & - & 1 & - & 1 & - & - \\
\hline Morchellaceae & - & 1 & 5 & 1 & - & - \\
\hline Pezizaceae & - & 2 & 1 & 1 & 1 & 1 \\
\hline Caloscyphaceae & 1 & 1 & 1 & - & - & - \\
\hline Discinaceae & 1 & 2 & - & - & - & - \\
\hline Pyronemataceae & 1 & 4 & 2 & 1 & - & - \\
\hline Diatrypaceae & - & 2 & - & - & 1 & - \\
\hline Xylariaceae & 1 & 2 & - & 1 & - & - \\
\hline Agaricaceae & 16 & 23 & 8 & 16 & 13 & 6 \\
\hline Albatrallaceae & - & - & - & 1 & & - \\
\hline Amanitaceae & 3 & 7 & - & 4 & 2 & 4 \\
\hline Hygrophoraceae & 3 & 5 & 2 & 2 & 6 & - \\
\hline Cortinariaceae & - & 6 & 4 & - & - & 3 \\
\hline Inocybaceae & 3 & 8 & 8 & 1 & - & 2 \\
\hline Marasmiaceae & 4 & 3 & - & 1 & - & - \\
\hline Entolomataceae & - & 1 & - & - & 1 & - \\
\hline Hydnangiaceae & - & 1 & - & - & - & - \\
\hline Lyophyllaceae & - & 2 & - & - & - & - \\
\hline Marasmiaceae & - & 3 & 1 & - & 2 & - \\
\hline Mycenaceae & 4 & 9 & 8 & 3 & 2 & - \\
\hline Physalacriaceae & 4 & 4 & 4 & 1 & - & - \\
\hline Pleurotaceae & 1 & 1 & 1 & 1 & - & 1 \\
\hline Pluteaceae & 1 & 1 & - & & - & 1 \\
\hline Psathyrellaceae & 2 & 9 & 4 & 7 & - & 3 \\
\hline Schizophyllaceae & 1 & 1 & 1 & - & 1 & 1 \\
\hline Strophariaceae & 4 & 16 & 10 & 4 & 3 & 3 \\
\hline Tricholomataceae & 11 & 22 & 16 & 11 & 9 & 7 \\
\hline Auriculariaceae & - & 5 & - & - & - & - \\
\hline Boletaceae & - & 4 & 1 & 7 & 2 & 6 \\
\hline Diplocystidiaceae & - & 1 & - & - & - & - \\
\hline Hygrophoropsidaceae & - & 1 & - & - & - & - \\
\hline Hymenogastraceae & - & - & - & - & - & 2 \\
\hline Omphalotaceae & - & - & - & - & - & 2 \\
\hline Gomphidiaceae & 2 & - & 2 & 1 & 1 & 1 \\
\hline Paxillaceae & 1 & & - & 1 & 1 & - \\
\hline Rhizopogonaceae & 1 & 1 & 1 & - & 2 & 1 \\
\hline Suillaceae & 1 & 3 & 1 & 2 & 3 & 1 \\
\hline Tapinellaceae & - & 1 & - & - & - & - \\
\hline Cantharallaceae & - & 1 & - & - & - & - \\
\hline Clavulinaceae & - & 1 & - & 1 & - & - \\
\hline Hydnaceae & - & 1 & - & - & 1 & - \\
\hline Dacrymycetaceae & 3 & 3 & 1 & 1 & - & - \\
\hline Geastraceae & 2 & 2 & 5 & 1 & - & 1 \\
\hline Gloeophyllaceae & 1 & 1 & 1 & 1 & - & - \\
\hline Clavariadelphaceae & 1 & 1 & - & - & 2 & - \\
\hline Gomphaceae & 1 & 2 & 2 & 1 & 2 & - \\
\hline Schizoporaceae & - & 1 & - & - & - & - \\
\hline Phallaceae & - & 2 & - & - & - & - \\
\hline Hymenochaetaceae & 2 & 2 & 3 & - & 1 & 3 \\
\hline Fomitopsidaceae & 5 & 5 & - & 1 & - & 1 \\
\hline Ganodermataceae & 3 & 4 & 1 & 2 & - & - \\
\hline Meruliaceae & - & 1 & - & $\frac{2}{1}$ & - & 1 \\
\hline
\end{tabular}


Tablo 2. Bölgede tespit edilen zehirli türler ve neden oldukları sendromlar(Bresinsky ve Besl,1990)

\begin{tabular}{|l|c|}
\hline \multicolumn{1}{|c|}{ Türler } & Sendrom \\
\hline Sarcosphaera coronaria & Gyromitra \\
\hline Amanita citrina & Phalloides \\
\hline Amanita gemmata & Pantherina \\
\hline Amanita pantherina & Pantherina \\
\hline Cortinarius rubellus & Orellanus \\
\hline Russula emetica & Gastrointestinal \\
\hline Russula nigricans & Gastrointestinal \\
\hline Hebeloma sinapizans & Gastrointestinal \\
\hline Omphalotus illudens & Gastrointestinal \\
\hline Boletus erythropus (Çiğ yendiğinde) & Gastrointestinal \\
\hline Boletus luridus (Çiğ yendiğinde) & Gastrointestinal \\
\hline Clitocybe dealbata & Muskarin \\
\hline Inocybe mixtilis & Muskarin \\
\hline Inocybe rimosa & Muskarin \\
\hline
\end{tabular}

\section{Kaynaklar}

Akata I., Çetin B., Işıloğlu M., Macrofungi of Ankara-Kızılcahamam Soğuksu National Park, Ot Sistematik Botanik Dergisi, 16:2, 177-188 (2009)

Akata I., Çetin B., Işıloğlu M., Macrofungal Diversity of Ilgaz Mountain National Park and Its Environs (Turkey), Mycotaxon, 113:287-290 (2010).

Breitenbach J., Kränzlın F., Fungi of Switzerland, Volume 1-5. Verlag Mykologia CH-6000 Luzern 9, Switzerland (1983-2005).

Bresinsky A., Besl H., A Colour Atlas of Poisonous Fungi, Wolfe Publishing Ltd., London (1990).

Dähncke R.M., 1200 Pilze, AT Verlag Aarau, Stuttgart (1993).

Doğan H.H., Kaşık G., Öztürk C., Aktaş S., A Checklist of Aphyllophorales of Turkey, Pakistan J. Bot., 37(2): $459-485$ (2007).

Ellis M.B., Ellis J.P., Fungi Without Gills (Hymenomycetes and Gasteromycetes), Chapman and Hill, London (1990). Jordan K., The New Guide to Mushrooms, Anness Publishing Ltd., Singapore (1996).

Kaşık G., Aktaş S., Öztürk C., Doğan H.H., Macrofungi Distribution of Gevne Valley, Mantar Dergisi/The Journal of Fungus, 1(2):25-32 (2010).

Köstekçi H., Yamaç M., Solak M.H., Macrofungi of Türkmenbaba Mountain (Eskişehir), Turk J. Bot., 29:409-416 (2005).

Kreisel H., Grundzüge Eines Natürlichen Systems der Pilze, Verlag Von J. Cramer, Stuttgart (1969).

Moser M., Keys to Agarics and Boleti, Gustav Fischer Verlag, Stuttgart (1983).

Phillips R., Mushrooms and Other Fungi of Great Britain and Europe, Pan Books Ltd., London (1981).

Servi H., Akata I., Çetin B., Macrofungal Diversity of Bolu Abant Nature Park (Turkey), African Journal of Biotechnology, 9(24):3622-3628 (2010).

Sesli, E., Denchev, C.M., Checklists of the myxomycetes, larger ascomycetes, and larger basdiomycetes in Turkey. 6th edn. Mycotaxon Checklists Online (2014). (http://www.mycotaxon.com/resources/ checkilsts/sesli-v106checklist.pdf): 1-136.

Watling R., Identification of The Larger Fungi, Hulton Educational Publications Ltd (1973).

Winkler R., 2000 Pilze Einfach Bestimmen, ATV. Aarau, Schweiz (1996). 